Proceedings of the 16th Czech and Slovak Conference on Magnetism, Košice, Slovakia, June 13-17, 2016

\title{
Scaling of Anhysteretic Curves for LaFeCoSi Alloy near the Transition Point
}

\author{
R. Gozdur ${ }^{a}$, K. Chwastek ${ }^{b, *}$, M. Najgebautr $^{b}$, M. Lebioda $^{c}$, E. Bernacki $^{a}$ \\ AND A. WODZYŃSKI ${ }^{b}$ \\ ${ }^{a}$ Department of Semiconductors and Optoelectronic Devices, Łódź University of Technology, \\ Wólczańska 211/215, 90-924 Łódź, Poland \\ ${ }^{b}$ Faculty of Electrical Engineering, Częstochowa University of Technology, \\ al. Armii Krajowej 17, 42-201 Częstochowa, Poland \\ ${ }^{c}$ Institute of Electrical Engineering Systems, Łódź University of Technology, \\ B. Stefanowskiego 18/22, 90-924 Łódź, Poland
}

\begin{abstract}
LaFeCoSi alloys have attracted much attention as they exhibit magnetocaloric effect in near room temperature. The paper focuses on the description of anhysteretic curve for LaFeCoSi alloy for temperatures close to transition point. For this purpose the Langevin function is applied. The paper confirms that the Langevin function might be a good starting point for development of analytical models aimed at examination of magnetic properties in La-based magnetocaloric materials.
\end{abstract}

DOI: 10.12693/APhysPolA.131.801

PACS/topics: 75.30.Sg, 75.60.-d, 75.60.Ej

\section{Introduction}

The growing interest of the scientific community in $\mathrm{La}(\mathrm{FeCoSi})_{x}, x=13$, compounds is due to their magnetocaloric effect (MCE) occurring in near room temperature and the possibility to tailor their properties by an appropriate modification of chemical composition and processing conditions [1-3]. Magnetocaloric devices are a competitive replacement for conventional vapour compression units as they do not use ozone depleting gases and they offer better efficiency. Room temperature magnetic refrigeration is thus perceived as an energy-saving technology which may reduce harmful burdens on the environment $[4,5]$.

The present paper is focused on the description of anhysteretic curves in $\mathrm{LaFe}_{10.8} \mathrm{Co}_{1.1} \mathrm{Si}_{1.1}$ compound for temperatures close to transition point. The anhysteretic state depends both on temperature and stress applied to or already present in the sample $[6,7]$. In the paper the effect of temperature on the shape of anhysteretic curve is considered.

It should be recalled that the concept of anhysteretic curve plays an important role in many descriptions of magnetization phenomena, as this curve is a crucial component of some hysteresis models [8-11].

\section{Experiment}

In the present study commercially available LaFeCoSi samples (four plates $36 \times 18 \times 5 \mathrm{~mm}^{3}$, total mass $93.435 \mathrm{~g}$ )

\footnotetext{
* corresponding author; e-mail: krzysztof . chwastek@gmail .com
}

were used. The application of bulk material made it possible to reduce the influence of micromagnetic defects and structural inhomogeneities on the actual magnetic properties of the examined samples. The Curie point of the investigated magnetocaloric compound was tailored up at $T_{\mathrm{C}}=300 \mathrm{~K}$. The peak changes of magnetic entropy were reached at $T_{\Delta S_{\max }}=296 \mathrm{~K}$. The alloy with nominal composition $\mathrm{LaFe}_{10.8} \mathrm{Co}_{1.1} \mathrm{Si}_{1.1}$ was used for further processing. The bulk samples were prepared by fine milling the $\mathrm{LaFe}_{10.8} \mathrm{Co}_{1.1} \mathrm{Si}_{1.1}$ powder, grain size below $5 \mu \mathrm{m}$ and by subsequent sintering at temperature above $1000^{\circ} \mathrm{C}$, applied pressure from the range $\langle 1.5,4\rangle \mathrm{t} / \mathrm{cm}^{2}$. The $\alpha$-Fe content at the beginning of crystallization was approximately $10 \%$ [12]. The composition was analyzed using an EDS probe X-MAX N80 from Oxford Instruments. Figure 1 depicts a fracture of the sintered microstructure.

The magnetocaloric material used in the study has a typical structure after HIP sintering process with a large numbers of defects, cracks and grain-boundaries. Nonetheless the micrographs confirm a high homogenization level of the sample with only a few micropores visible on the fractured surface. Further EDS elemental quantitative analysis of structural defects marked as 22,23 , and 24 indicated the presence of impurities. Slightly different compositions were observed in the black spots (cf. Fig. 1). The measured composition in the spot 23 suggested a local existence of lanthanum oxide whereas the spot 24 pointed out the nucleation of the $\alpha$-Fe phase. The analysis of composition from rectangular spread 21 proved a good consistency with the nominal composition (Fig. 2). The measurements and calculations of percentage weights are collected in Table I. Thermal dependences of magnetic polarization $J(T)$, coercive field $H_{c}(T)$, power loss density $P(T)$, hysteresis loops $J(H)$ and ini- 


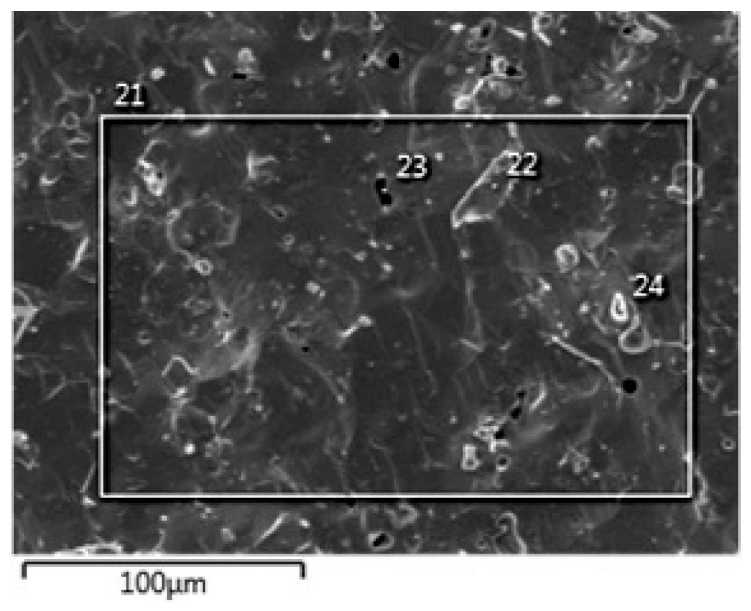

Fig. 1. SEM micrograph of a typical fracture of sintered LaFeCoSi sample.

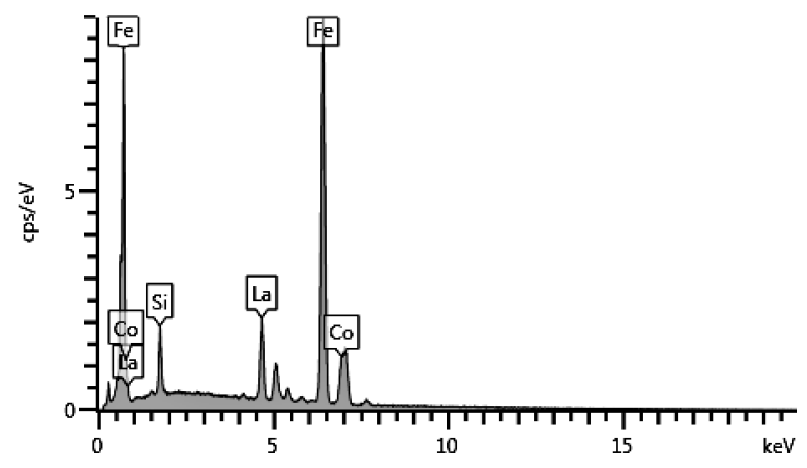

Fig. 2. The EDS spectrum of $\mathrm{LaFe}_{10.8} \mathrm{Co}_{1.1} \mathrm{Si}_{1.1}$ structure obtained for spread $21\left(220 \times 150 \mu \mathrm{m}^{2}\right)$.

tial magnetization curves $J(H)$ were measured in order to verify the hypothesis of scaling of anhysteretic curves. The magnetic properties and power losses were measured in accordance with the recommendations of IEC 60404 standards. The closed magnetic core was assembled from four LaFeCoSi plates. Magnetic field strength $H$ and polarization $J$ were measured using a shunt resistor and pick-up coils, respectively. The temperature dependences of power loss density were determined pri-

TABLE I

The nominal and the measured LaFeCoSi composition, wt.\%.

\begin{tabular}{c|c|c|c|c|c}
\hline \hline \multirow{2}{*}{ Element } & \multirow{2}{*}{ Nominal } & \multicolumn{4}{|c}{ Spread } \\
\cline { 3 - 6 } & & 21 & 22 & 23 & 24 \\
\hline $\mathrm{O}$ & - & - & - & 0.55 & - \\
$\mathrm{Si}$ & 3.69 & 3.61 & 1.71 & 0.68 & 1.43 \\
$\mathrm{Fe}$ & 71.99 & 68.73 & 71.02 & 69.49 & 85.05 \\
$\mathrm{Co}$ & 7.74 & 9.21 & 9.37 & 8.94 & 8.41 \\
$\mathrm{La}$ & 16.58 & 18.46 & 17.91 & 20.33 & 5.11 \\
Total: & 100.0 & 100.0 & 100.00 & 100.0 & 100.0
\end{tabular}

marily from $J(H)$ loops using averaging of the Poynting vector over a cycle of the excitation field. In order to reduce the heating of the samples due to eddy currents, the excitation frequency was kept at $1 \mathrm{~Hz}$. Due to extremally low values of power loss density above the transition point, the measurements were verified using the unbalanced bridge method [13]. The magnetic sample were tested in a thermostatic chamber with controlled temperature $(\Delta T=0.2 \mathrm{~K})$. The experimental results, curves and the detailed description of the measurement setup have been discussed previously in detail in Refs. [14, 15].

\section{Results}

The anhysteretic curves were obtained after postprocessing the experimental hysteresis $M-H$ dependencies, by taking the middle curve between ascending and descending loop branches [16]. The reconstructed $M_{a n}(H)$ dependences were fitted with the Langevin function

$$
M_{a n}=M_{s}(T)\left[\operatorname{coth} \frac{H}{a(T)}-\frac{a(T)}{H}\right] .
$$

The shape parameter of the Langevin function $a(T)$ is directly proportional to temperature. Therefore plotting the reduced magnetization $m=M_{a n} / M_{s}$ vs. the ratio $H / T$ should yield a universal dependence, valid both below the transition point as well as in the paramagnetic regime $[17,18]$. The modelling results are shown in Figs. 3 and 4 . As it can be seen from Fig. 3 there are slight deviations between the obtained trends for higher magnetization values. They may be attributed to fluctuations affecting the measurement results at temperatures close to the Curie point [19].

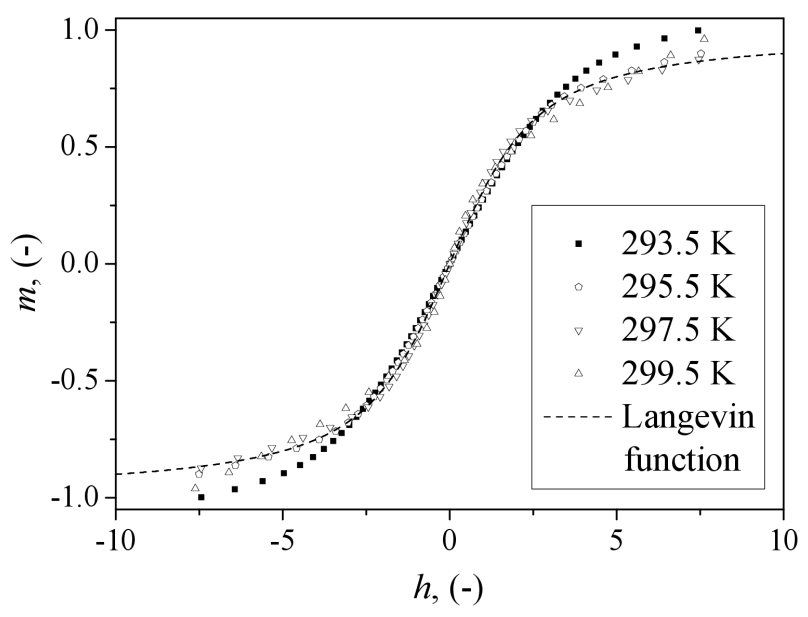

Fig. 3. Reduced anhysteretic magnetization vs. reduced magnetic field for temperatures below the transition point.

During fitting the saturation magnetization $M_{s}$ was treated as a free parameter, dependent on the instant value of temperature. It was found that the obtained trend of $M_{s}(T)$ dependence is to some extent similar to 


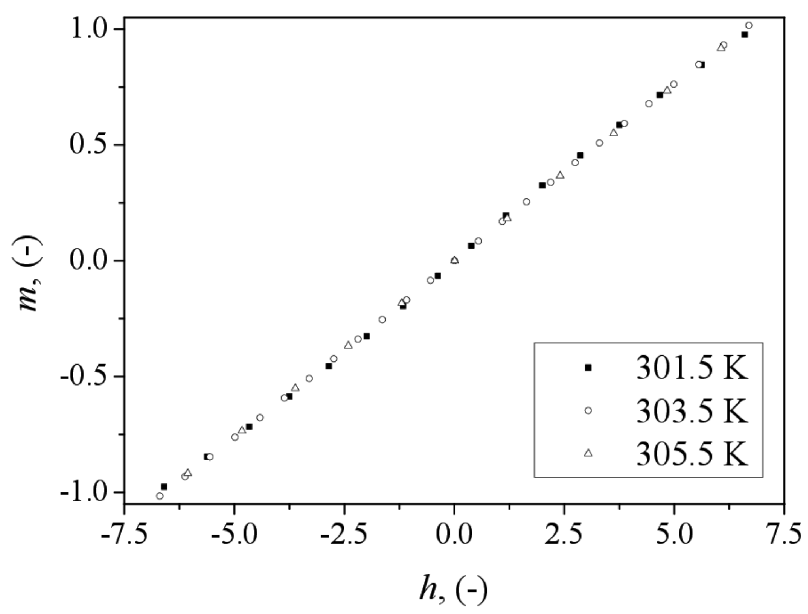

Fig. 4. As in Fig. 3 but for temperatures above the transition point.

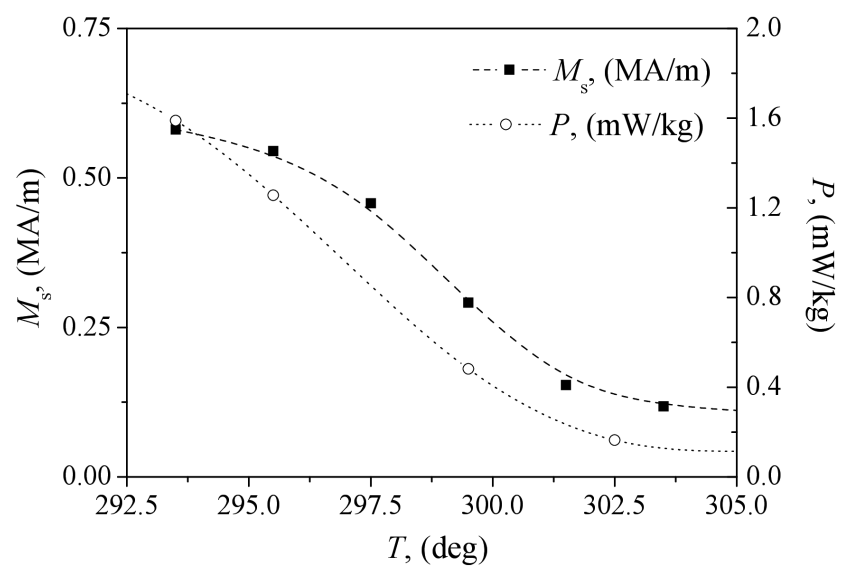

Fig. 5. The trends for $M_{s}$ and measured loss density $P$ vs. temperature.

the trend of power loss density vs. temperature $P(T)$ dependence, cf. Fig. 5.

\section{Conclusions}

In the paper the dependence of anhysteretic magnetization vs. temperature in LaFeCoSi alloy was described with the Langevin function. It was found that the description may be adequate both for temperatures below and above the transition point. The fitting results suggest that there might be a correlation between the values of saturation magnetization and power loss density.

\section{References}

[1] P. Gębara, P. Pawlik, I. Škorvánek, J. Marcin, J.J. Wysłocki, Acta Phys. Pol. A 118, 910 (2010).

[2] J. Liu, J.D. Moore, K.P. Skokov, M. Krautz, K. Löwe, A. Barcza, M. Katter, O. Gutfleisch, Scr. Mater. 67, 584 (2012).

[3] R. M'nassri, A. Cheikrouhou, J. Supercond. Nov. Mater. 27, 1059 (2014).

[4] K.A. Gschneidner, Jr., V.K. Pecharsky, Int. J. Refrig. 31, 945 (2008).

[5] K.G. Sandeman, Scr. Mater. 67, 566 (2012).

[6] M.J. Sablik, R.A. Langman, J. Appl. Phys. 79, 6134 (1996).

[7] B. Kvasnica, F. Kundracík, J. Magn. Magn. Mater. 162, 43 (1996).

[8] D.C. Jiles, D.L. Atherton, J. Magn. Magn. Mater. 61, 48 (1986).

[9] R.G. Harrison, IEEE Trans. Magn. 39, 950 (2003).

[10] J. Takács, Physica B 372, 57 (2006).

[11] C.S. Schneider, IEEE Trans. Magn. 48, 3371 (2012).

[12] M. Katter, V. Zellmann, G.W. Reppel, K. Uestuener, IEEE Trans. Magn. 44, 3044 (2008).

[13] R. Gozdur, A. Majocha, Przegl. Elektrotechn. 4, 79 (2010).

[14] R. Gozdur, M. Lebioda, Ł. Bernacki, Acta Phys. Pol. A 128, 98 (2015).

[15] R. Gozdur, M. Najgebauer, J. Electr. Eng. 66, 37 (2015).

[16] J. Krah, A. Bergqvist, Physica B 343, 35 (2004).

[17] P. Allia, M. Coïsson, P. Tiberto, F. Vinai, M. Knobel, M.A. Novak, W.C. Nunes, Phys. Rev. B 64, 144420 (2001).

[18] P. Allia, M. Coïsson, P. Tiberto, F. Vinai, J. Magn. Magn. Mater. 226-230, 1904 (2001).

[19] L.E. Reichl, A Modern Course in Statistical Physics, Wiley, New York 1998. 